\title{
The Impact of Computers Design on the Traditional Art Design
}

\author{
Yonghua Jing \\ Minsheng College \\ Henan University \\ Kaifeng 475004 China
}

\begin{abstract}
Due to advances in computer technology, interactive computer graphics applications are evolving, and art computer technology is integrated into the design, making science and art in many fields fused. Art is a computer up to date to explore the area and become industry's hottest hotspot art and design, traditional art and design are a breakthrough and innovation development. The article analyzes the application of computer technology in art design.
\end{abstract}

Keywords- Science; Arts; Computer; Art and Design

\section{INTRODUCTION}

In today's increasingly competitive society, arts education has quality education, the traditional design arts education cannot meet the needs of the new era fast-paced, high-quality work, study and design of the 21 st century that is an era of information and science, the information age is how to train professionals in the design. The computer art design with outstanding design work and computer art design is necessary for social progress, is the need of the times, and is the need of social development. Computer Art and Design education is the inevitable trend of technological development, and is a combination of technology and information science education. As an advanced computer design tools, it is not only the sound and informative map, showing a convenient, fast, vivid images, which has a strong sense of form and vivid special effects that are beyond the reach of traditional design results. In addition, it can modify the new computer science students of art and design, new knowledge is very interested in learning this knowledge, learning motivation large, high enthusiasm in the design arts education in the new era it is indeed an indispensable discipline. It is only to traditional design and computer art design arts education organically to combine education, in order to adapt to the new era of education reform and opening up. There is the need to develop, and the traditional design art education is a basic course, it is also an indispensable basis for discipline, it can put the traditional design and modern technology in the design and use of reasonable use education, in order to design education to a new level.

\section{THE FUtURE OF THE COMPUTER ART DESIGN EDUCATION}

In the development of human society today, between technology and art changes, we are faced with a new problem, every human technological progress will bring great changes in art of computer art design education in the new issue. It is the arrival of the 21 st century; the traditional design education cannot meet the new era of reform and development needs. The computer art design is to create visual art that is not only wealth, but also on the social culture of visual thinking and research. the traditional design of basic education cannot be ignored, to master modern new technology (computer graphic design software) that should be given more attention. The rapid development of new software meets the needs of the new era of reform, development, and times are ahead, society continues to progress, development and social needs far exceed the speed of talents education to go in the era precedent, training for the new era of art and design talent needs. From the early 1990s the computer industry booms, computer development today has entered the field of art and design education, the use of design education of computer art, greatly changed people's thinking and ideas to improve the understanding. From laser typesetting technology, computer inkjet visual has effects of art printing 3D animation titles and advertising, multimedia art, animation, rendering indoor and outdoor environment design, graphic design. And so the effect of construction plans are computer generated art, effects processing picture image, outdoor, newspapers magazine advertising, interior design and other visual language, computer art design today has entered our daily lives, it greatly improves and enhance the quality of life, affecting the adjustment of social structure, and a strong impetus to the development of society .

\section{COMPUTER ART DiFFERENT FROM TRADITIONAL ART UNIQUE ADVANTAGES}

A. Impact of the computer generation and development on human society and on the productivity

Computer is a great invention of the humankind, big fast computing speed, workload, it can be completed in the field, 
there are many people's mental and physical labor that is unmatched, as an advanced production tools, widely used in computers in every field of human production processes plays a significant role, which is an important tool to improve productivity.

\section{B. The Impact of the Art Computer on the Art, Including the Impact of Changes in the Way the Creation and Theory}

The emergence of computer has important changes on art field of art in the creation and dissemination methods. First, the techniques (including painting and graphic design, mainly art design), traditional techniques in many complex techniques are simplified and the precise size control. For example, in the field of space design renderings, drawing and building structure diagrams use auto desk company's common computer-aided design software Autocad, 3D video animation software MAYA, generic 3D modeling and animation software 3Dsmax. Autocad has considerable extent out of the traditional ruler and drawing tools that generated a lot of labor, saved a lot of time. The architectural renderings field can be used in conjunction with Autocad and 3Dsmax modeling, rendering performance chart; prepress in graphic design, especially the design of these areas need to achieve size precision design, through graphic design software such as Illustrator, Corel-draw, Photoshop and other print and output that are combined with precision equipment, and they can be done accurately. Effect has the control of the degree of freedom: a variety of mature design and painting software provides a complete, high degree of freedom of adjustment programs, through color, light and shade, such as picture adjustment. You can always change the face of the work, without the need to re-coloring; modification is so easy, undo has been carried out beyond the traditional techniques that are unique art computer techniques in traditional art. Many mistakes will lead to naught working function so that the waste rate is greatly reduced; we should use time and effort to save the artwork.

With the increasing popularity of computer technology and the Internet, the corresponding design industry has also been some form of innovation and development. Modern design is closely related to people's lives, to adapt to the market economy and the development of computer design practical need. Art is no longer simply a designer with a brush and drawing paper for drawing, but with a computer to show it, compared with the traditional art design, computer design is to promote the formation and development of the visual design of a series of related industries. Computer art design achieved with innovative tools for creative art design tools, in the traditional art design based on the development of computer technology and continuous improvement.

Computer art is the combination of computer and art is the crystallization of technology and art. The rise of computer art is making art more diverse performance practices, especially in the application of design works of art; the use of computer technology has been quite common. Computer art opened up new areas of art and design, and the rapid penetration of the traditional art fields. Now, from graphic design to arts and crafts, advertising posters are from design to design book covers, from film and animation to movie special effects, from website design to industrial design, from professional to amateur art creative arts entry, one can see traces of computer art. The process of advertising, decoration, modeling, and other creative arts, and it will be more closely integrated with computer art. In future development, graphic designers do not understand computer technology to display their artistic talents of space that will be gradually reduced, their situation will be like a new artist that does not get the same brush. Therefore, to promote the basics of computer art is our obligation related to professional educators.

\section{TRaditional ARt Still Has Its IrRePlaceable POSITION}

The principle of the contents of computer art is from traditional art, despite the long-term accumulation of computer art to the art in the field of development that has brought great changes. Nevertheless, the core of computer art is still art; dismantling "computer art", the term can be understood to do "in order to computer aided technology as art, "so traditional art theory in the field of computer art is still irreplaceable. A good painting or design in computer art workers, in addition to his need to have skilled software operating skills required to be aesthetic capacity required by traditional art, creative thinking, modeling ability, cognitive ability. By learning the traditional art education perspective, structure, lighting, composition, form, color, anatomy, art history and Western theories of knowledge in the field of computer art still has the traditional art field in almost the same importance. 2.2 traditional art techniques have profound impact on the traditional art techniques of computer art that is art workers after many practice lessons learned. In general, there are two main aspects, one is the ability to deal with the picture or art reproduction, the second is the creation tools and materials (such as various paper and pencil) understanding and operating performance. These techniques require a master art worker spend a lot of training and labor, and these experiences will be used, directly or indirectly, in computer art fields.

\section{A. Unique Advantages of Computer Art Design}

As an advanced computer design tool, it can not only sound and picture, information storage capacity displays convenient, vivid images, dynamic effects are beyond the reach of conventional design, and they can modify it especially convenient. As an advanced production tool, it is widely used in computer production in all areas of human life aspects that played a huge role, a substantial increase in production efficiency. It gives designers who have left more time for reflection and design, imagination has been expanded by the graphic design, three-dimensional design, and web design and animation film and television production. 


\section{B. Close to Computer Art Design and Traditional Art Designs}

Despite the development of computer art design to graphic design field, it has brought great changes, but the core remains the computer art design of art design. The reason is why today's art design flourishes, mainly due to functional design work, artistic and cultural taste. This requires that the designer has a good aesthetic ability and innovative thinking, and continue to inject art and culture of gold for the design work. So an excellent computer graphic designer, he needs to have in addition to skilled software skills, but also has a good foundation of traditional art and design. If you do not have the computer operations only traditional art design basis. You cannot create works of art with artistic line; it really wants to create high-quality computer art design work, but also requires a computer graphic design and traditional art design organically up designer aesthetic ability is stronger, the higher artistic accomplishment, the higher their design works out the taste, the more it can get popular.

\section{COMPUter ART DeVelopment Has DePENDENCY ON THE FIELD OF THE DEVELOPMENT OF COMPUTER TECHNOLOGY}

Nowadays, traditional art has no need to break the technical barriers, with the development of computer art of computer technology that continue to develop. Development of computer art and computer technology are mutually interdependent, three important trends in the future development of computer technology has better performance, a higher degree of intelligence, and computer penetration in the wider human society, these trends of the development of computer art is also exerting a profound impact. Computer art computer hardware performance has requirements for very high performance graphics workstation performance that is usually much higher than in other areas; obvious graphics processing speed can be raised to a higher performance computer course, computer performance is busy business and art animation staff tense game production company, which is not enough. They will need to update faster computer to do more work in a short time scene rendering and rendering. To improve computer performance will require a computer at the same time to be a more complex system of higher degree of simulation scenarios and models. Input and output devices are in addition to the speed of the computer performance art focused on requirement. The output device is what you want to focus on highperformance is the professional cathode ray tube display; it has true professional printers, publishing in the field of offset printing equipment and processing technology. Sampling is really art computer input device requirements, such as highresolution digital sampling drawing board, it is very close to the sample to write the true strength and direction of the brush; and it obtains material vital equipment that also has a high-performance scanners and digital cameras. It should improve work efficiency and quality of work is inseparable from the development of these high-performance peripherals.

While computing the development of artificial intelligence level is close to art workers and signs of creative and unique thinking in the field of art that we have not yet been found. The actual stage art workers need is the software development that has become more humane. Art workers are looking for software developers, which is the software easier to use and more efficient to use, the effect is more abundant, easier to control. Computer in human society is highly permeable expanding the scope of application of mobile gaming. These non-general-purpose computers are expanding the exhibition space of computer art works that also provide it with more media types, but it is also to expand the application fields of computer art development.

\section{CONCLUSIONS}

Traditional art design and computer image processing technology are combined with each other to produce a mastery of computer art design, art design is the creation of a higher capacity requirements, while also significantly reducing design effort and expanding the art of creative space of an impact. It is the direction of the trend of the times but also become an essential tool for professional designer's work of art. Meanwhile it is today's trend of the times; we have the ability to develop good aesthetic and artistic creative foundation to focus on more art with traditional designs.

\section{Reference}

[1] Qi Min. Contrast with traditional computer art design art design analysis [J] electronic test, 2014,16: 112-113 + 84 .

[2] Liu Xiaoni. Science and art - computer art design [J] electronic test, 2014,19: 105-107.

[3] Cheng Nan. Computer art design and the traditional art design [J] young writer, 2011,06: 94.

[4] Zhao Han. Rrelationship development computer art and traditional art [J] Computer Knowledge and Technology (Academic Exchange), 2007,12: 1708-1709.

[5] Wang Tianqi. Combination of science and art - computer art design [J] Shanghai packaging, 2007,10: 21-23.

[6] Xiao Zhongwen. Computer art design professional construction and development of [J] Hunan packaging, 2001, 01: 25-26.

[7] Gao Mingchun. Popularization of computer art design [J] Silk, 1995,03: 42-44. 